Hygiene in der Zahnarztpraxis (inkl. Begehungen nach MPG) Teil 2

(Bitte beachten Sie auch den Kurs 13132)

Termin: 4.12.2013

Referent:

Dr. J. Szafra niak, Viersen

Kurs-Nr.: $13133 \mathrm{~T}$

Gebühr:

170,-€, Praxismitarbeiter (ZFA) $90 €$

Zahnersatz - Abrechnung nach BEMA und GOZ - Teil 4 Seminar für Zahnärzte innen und Praxismitarbeiter/innen

Termin:

Referenten:

13. 12. 2013

ZA M, Hendges, Köln; ZA L. Marquardt, Krefeld

Kurs-Nr:

Gebühr: $\quad 30,-€$
Innovative Konzepte zur vorhersagbaren Deckung singulärer und multipler Rezessionen

Termin:

14. 12. 2013

Referent:

Prof. A.

Sculean, Bern

Kurs-Nr.: $\quad 13127$

Gebühr: $\quad 320,-€$

Kofferdam in

100 Sekunden

Termin:

14. 12.2013

Referent:

Dr. J. Müller, Wörth a.d.

Isar

Kurs-Nr.:

13126

Gebühr:

$260 €$, Praxismitarbeite

(ZFA) $130,-€$

Information und Anmel-

dung:

Zahnärztliche Kurse im KarlHäupl-Institut der Zahnärztekammer Nordrhein,

Emanuel-Leutze-Str. 8 40547 Düsseldorf

\title{
Für Kurzentschlossene
}

13. (Nord)Deutsches CMD-Curriculum startet im November auf Sylt

Block I (CMD-Diagnostik): 9.-13.11.2013

Block II (CMD-Therapie): 15.-19.2.2014

Zertifizierungsprüfung: 22.-23.11.2014

Zum 13. Mal laden Dr. med. dent. Christian Köneke (CMDSpezialist, Bremen) und Gert Groot Landeweer (Physiotherapeut, Gundelfingen) interessierte Kollegen zum (Nord) Deutschen CMD-Curriculum nach Sylt ein.

Der 1. 5-Tages-Block vom 9.-13. November 2013 beschäftigt sich ausschließlich mit der Diagnostik der CMD. Es werden alle dazu notwendigen Techniken vermittelt und ausgiebig geübt. Die Kurskernzeit ist täglich von 8-15 Uhr. Nach Absolvieren des Kurses sollte jeder Teilnehmer alle Handgriffe, die zur Diagnostik notwendig sind, sicher beherrschen.

Der 2. 5-Tages-Block widmet sich der CMD-Therapie und findet vom 15.-19. Februar 2014 statt. Ziel sei es, so Köneke, dass alle Teilnehmer die Fähigkeit entwickeln, aus der gewonnenen Diagnose auch eine sinnvolle Therapie abzuleiten. Schienen unterschiedlicher Arten werden mit ihren jeweiligen Indikationen und Kontraindikationen vorgestellt. Zudem werden als mögliche Optionen im Anschluss an eine erfolgreiche CMD-Therapie Prothetik, Implantologie und Kieferorthopädie ausführlich behandelt. Weil die Kurse auch für ärztliche Kollegen und Physiotherapeuten zugänglich sind, fließen auch fachübergreifende Behandlungskonzepte in den Kurs mit ein.

Ende 2014 wird es auf Sylt die Möglichkeit der Zertifizierungsprüfung geben. Das Curriculum ist als strukturierte Fortbildungsveranstaltung von den Zahnärztekammern anerkannt und endet mit der Bezeichnung „Zertifizierter CMD-Therapeut (NCC)“. Die Zertifizierung kann als Wissensnachweis für die Erlangung des Tätigkeitsschwerpunktes „CMD-Diagnostik und -Therapie“ verwendet werden. Fortbildungspunkte: 135

Informationen und Anmeldung:

PT Gert Groot Landeweer und Dr. Christian Köneke Upledger-Institut Deutschland

Gutenbergstraße 1 Eingang C, 23611 Bad Schwartau

Telefon: 0451-479950, Telefax: 0451-4799515

E-Mail: info@upledger.de, Internet: www.cmd-therapie.de 